\title{
Biplanar Open-Wedge High Tibial Osteotomy with Locking Plate for Treatment of Osteoarthritic Varus Knee
}

\author{
Bahaa Kornah'1, Said K. Abdel-Hameed ${ }^{2}$, Mohamed Abdel-AAl ${ }^{3}$ \\ ${ }^{1}$ Faculty of Medicine for Boys, Al-Azhar University, Cairo, Egypt \\ ${ }^{2}$ Faculty of Medicine for Girls, Al-Azhar University, Cairo, Egypt \\ ${ }^{3}$ Manshet El Bakey General Hospital, Ministry of Health, Cairo, Egypt \\ Email: btmnail2010@hotmail.com
}

How to cite this paper: Kornah, B., Abdel-Hameed, S.K. and Abdel-AAl, M. (2019) Biplanar Open-Wedge High Tibial Osteotomy with Locking Plate for Treatment of Osteoarthritic Varus Knee. Open Journal of Orthopedics, 9, 1-13.

https://doi.org/10.4236/ojo.2019.91001

Received: September 14, 2018

Accepted: January 8, 2019

Published: January 11, 2019

Copyright $\odot 2019$ by author(s) and Scientific Research Publishing Inc. This work is licensed under the Creative Commons Attribution International License (CC BY 4.0).

http://creativecommons.org/licenses/by/4.0/

\begin{abstract}
Aim: To introduce and characterize the modified biplanar opening high tibial osteotomy with rigid fixation to treat unicompartmental knee arthritis with varus deformity in active lifestyle patients. Patients and Method: 48 patients (19 females) with monocompartmental gonarthrosis of the knee combined with varus malalignment, 41 with bilateral affection and 7 cases with unilateral gonarthritis (total 89 knees). Mean age: 53 years (range from 42 to 61 years). They were treated and followed from June 2010 to May 2014 (35 months). Biplanar opening high tibial osteotomy technique was used and fixed by low profile locked T-plate $(21$ cases) and low profile anatomical proximal tibial plate (27cases). Pre-operative mean varus deformity was $16.5^{\circ}$ $\left(9^{\circ}-19^{\circ}\right)$. We utilized Lysholm score and Knee Society Score for evaluation of preoperative and follow-up data. Results: The average correction angle was $11.50 \pm 2.50^{\circ}\left(7^{\circ}-19^{\circ}\right)$. Preoperative posterior tibial slope was $8.04^{\circ} \pm 1.30^{\circ}$ and at last follow-up $9.15^{\circ} \pm 1.60^{\circ}$, respectively $(\mathrm{p}=0.437)$. The joint space distance increased from $4.05 \pm 1.30 \mathrm{~mm}$ to $4.83 \pm 1.33 \mathrm{~mm}(\mathrm{p}<0.001)$. The average time to complete bone union was $14.69 \pm 1.5$ weeks. There were no cases of delayed union or non-union. No major complications like broken plate, nerve injury, or blood vessel injury occurred. No progression of degenerations developed in the three compartments of the knee at final follow-up. The mean score on the Lysholm-Gillquist knee functional scoring scale was $45.5 \pm 21.7$ preoperatively, and it improved to $77.0 \pm 23.9(\mathrm{p}<0.001)$. There was no obvious difference in the range of motion before and after operation. The average knee score improved from $51.19 \pm 11.82$ to $93.49 \pm 5.10$. Conclusion: Biplanar opening high tibial osteotomy fixed with locking compression
\end{abstract}


plate gives good results for symptomatic unicompartmental gonarthrosis with genu varum. Also this technique can be applied for medial compartment degeneration of the knee without deformity with good functional outcomes.

\section{Keywords}

Osteotomy, Biplanar, Varus, Osteoarthritis, Locked Plate

\section{Introduction}

Knee osteoarthritis is viewed as a standout amongst the most widely recognized musculoskeletal disorders in charge of awesome disability especially in old patients and sometimes in youthful dynamic patients. It constitutes a noteworthy issue as it influences ordinary every day activities of person. Limb malalignment with varus deformation particularly in unicompartmental gonarthrosis is viewed as major contributing element in this age group. Great conflicts in regards to careful treatment had been issued long time ago. Transferring load from diseased medial joint compartment to the more sound lateral one was the thought behind high tibial osteotomy issue so; halting progression of the disease and avoiding or postponing total knee arthroplasty [1]. The idea started in German literature in the 19th century (Langenbeck, B. 1854) [2], and became popularized and accepted technique after (Coventry, M. B.) [3] who made the osteotomy proximal to the tuberosity, with the advantage of faster healing. With the advent of total knee arthroplsty, the inquiries of its cost, long term results, life span of prosthesis, the higher infection rate and multiresistance of microscopic organisms had been cautioned. As of now, the normal future is getting longer and longer in cutting edge countries so; numerous creators thought about high tibial osteotomy as brief medical procedure until knee arthroplasty. This has no negative importance if the brief medical procedure gives help with discomfort of over 10 years, at that point it could give numerous advantages to patients. Variable systems and changes of high tibial osteotomy had been developed. Coventry [3] began by short wedge osteotomy which was not without a few deficiencies including: damage to peroneal nerve inferable from utilization of parallel approach, obligatory fibular osteotomy, plausibility of compartment syndrome, limb shortening with marked correction and difficult achievement of arthroplasty owing to lateral offset of proximal tibia. Open wedge osteotomy had been preferred and becoming increasingly popular for the treatment of varus gonarthrosis in the active patient to overcome previous shortcomings and the theoretical, easier subsequent total knee replacement (TKR) compared with closed wedge high tibial osteotomy CWHTO [4]. Modification of the OWHTO technique has been advised later on by many authors and we follow the recommendations of Kim et al., [5] utilizing biplanar open wedge high tibial osteotomy BPOWHTO and accordingly, the present study was undertaken to evaluate the 
clinical, functional outcomes and complications occurring after biplanar medial open wedge BPOWHTO in medial osteoarthritis of the knee joint with varus deformity in a prospective case series of 48 patients.

\section{Patients and Method}

Our Case series prospective study involved 48 patients (19 females and 29 males) with monocompartmental degeneration of the knee combined with varus malalignment of the leg less than grade III (Ahlback classification) [6]. 41 cases had bilateral affection while 7 cases had unilateral affection (total 89 knees). They were treated and followed from June 2010 to May 2014 (49 months) with mean follow-up duration 25.6 months (range, 19 to 35 months). They underwent Biplanar opening wedge high tibial osteotomy technique and fixed with low profile locked T-plate (21 cases) or low profile anatomical proximal tibial locked plate (27 case). There was no specific guideline to decide which plate would be applied. Inclusion criteria included symptomatic medial osteoarthritis of the knee joint, varus limb alignment, and an intact lateral joint compartment. Exclusion criteria involved: patients with history of infection, overweighted patients, bicompartmental, rheumatoid arthritis, history of proximal tibial fractures, severe varus deformity, range of knee motion $<90^{\circ}$, any vascular or neurological impairment of the limb, recurrent cases of high tibial osteotomy and patients refusing to sign informed consent. Age ranges from 42 to 61 years. Pre-operative mean varus deformity was $16.5^{\circ}\left(9^{\circ}-19^{\circ}\right)$. Generally, $3^{\circ}$ to $6^{\circ}$ of valgus overcorrection was planned preoperatively, as described by Dugdale et al. [7] (Figure 1). For younger patients ( $<50$ years) with minimal degeneration of the medial compartment, correction to a neutral mechanical axis was planned.

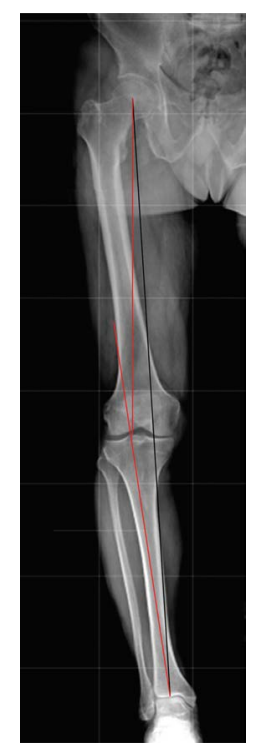

Figure 1. Preoperative planning on anteroposterior standing long-leg radiographs. Slight valgus overcorrection $\left(3^{\circ}-6^{\circ}\right)$ of valgus, $62.5 \%$ of the lateral tibial plateau) was planned [7]. 
The study was initiated after receiving approval from the institutional ethics committee for research in accordance with the ethical standards laid down in the 1964 declaration of Helsinki and its later amendments. Also, a written consent had been obtained from the patient for participating in the study.

Final evaluations were performed using the preoperative and follow-up data for: Range of knee motions, Lysholm Gillquist score [8], Knee society score, average deformity correction angle, posterior tibial slope change, joint space distance, average time to complete bone union and development of complications.

The statistical analysis of results was done using SPSS ver. 19 and statistical significance was accepted for $\mathrm{p}$-values $<0.05$.

\section{Preoperative planning:}

Through clinical examination for all patients to detect any medical or surgical comorbidity that may affect study outcomes, MRI was done to exclude cases with bicompartmental gonarthrosis. Radiographs of the whole limb should include posteroanterior and lateral weight-bearing radiographs and the knee joint were in full extension. The degree of varus deformity was detected. Target correction angle was measured at the point where the mechanical axis of the lower limb passed through the Fujisawa point [9], which is $62.5 \%$ from the medial tibial articular margin (Figure 2).

\section{Surgical techniques:}

The patient was placed supine on the operating table, 40 cases operated upon under spinal lumber anesthesia while 8 cases under general anesthesia. Bilateral knee examination under anesthesia was performed. A well padded high thigh tourniquet applied and draping the patient limb and C-Arm to check correct visualization of knee joint in both planes done, oblique incision done extending from joint line to medial aspect of tibial tuberosity (Figure 3). The periosteum detached from tibia with retraction of anserine tendon inferiorly (Figure 4).

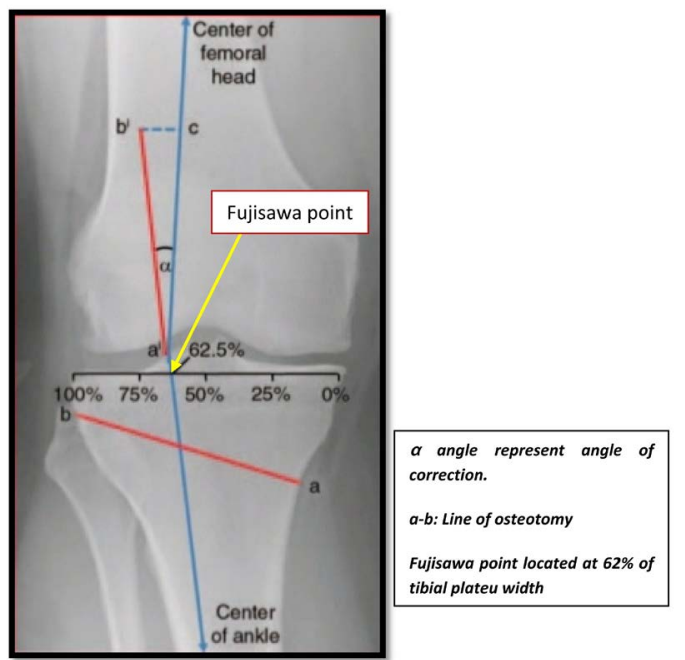

Figure 2. Fujisawa point: Fujisawa, Y., Masuhara, K., Shiomi, S.: The effect of high tibial osteotomy on osteoarthritis of the knee. An arthroscopic study of 54 knee joints. Source: [9]. 


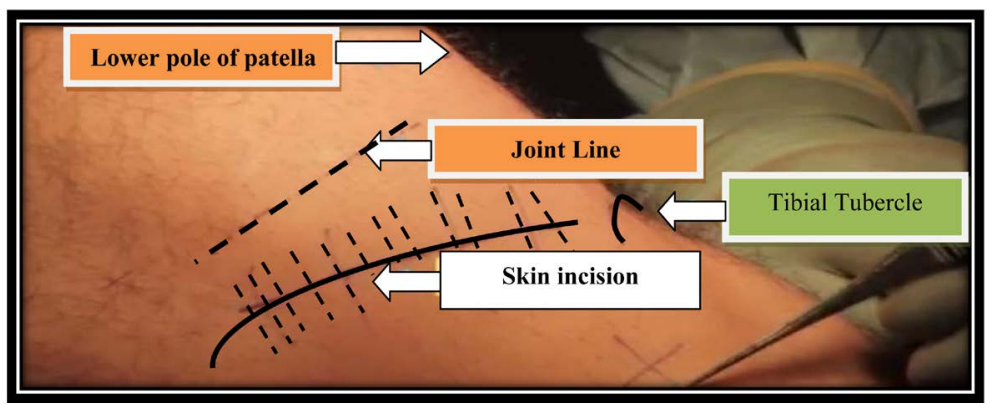

Figure 3. Approach.

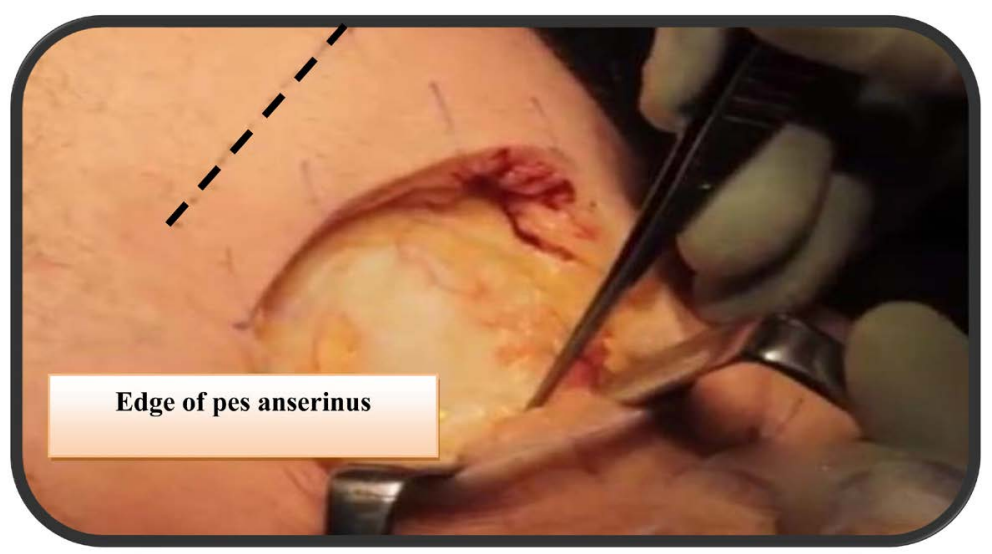

Figure 4. The periosteum detached from tibia with retraction of anserine tendon inferiorly.

A retractor placed posteriorly to protect neurovascular bundle and another one retracting patellar tendon (Figure 5).

Under image control 2 parallel K-wires $(2 \mathrm{~mm}$ ) placed $4 \mathrm{~cm}$ below the medial joint line passed obliquely from medial border of tibia directed toward the tibio-fibuler joint (15 mm from tip of fibula) (Figure 6). Two osteotomies was done along the medial surface of proximal tibia. The first osteotomy cut run horizontally oblique parallel to the tibial slope targeting the tip of the fibular head, and the second osteotomy run oblique coronal at $130^{\circ}$ to the horizontal oblique osteotomy leaving about $10 \mathrm{~mm}$ of lateral tibial cortex intact (Figure 7).

A laminar spreader introduced into osteotomy site opening the bone gradually and left in place for 5 minutes to allow for stress relaxation of the cortices and prevent fracture. until the correct angle desired reached. Assessment of the limb alignment had been done under C-Arm control using diathermy cable passing through hip, knee, and ankle and through Fujisawa point (Figure 8). Readjustment of osteotomy opening precisely by opening or closing the gap gradually. so that the cable line passing through $62 \%-66 \%$ of tibial width and posterior tibial slope readjusted to increase stability of joint in extension. Locking compression T-plate (21 cases) and anatomical proximal tibial plate (27 cases) applied on antero-medial tibial surface fixing the osteotomy. The size of the plate depended basically on 2 factors: the required correction angle and the size of the patient's tibia. At first, the proximal bone segment fixed and a lag screw was then inserted 


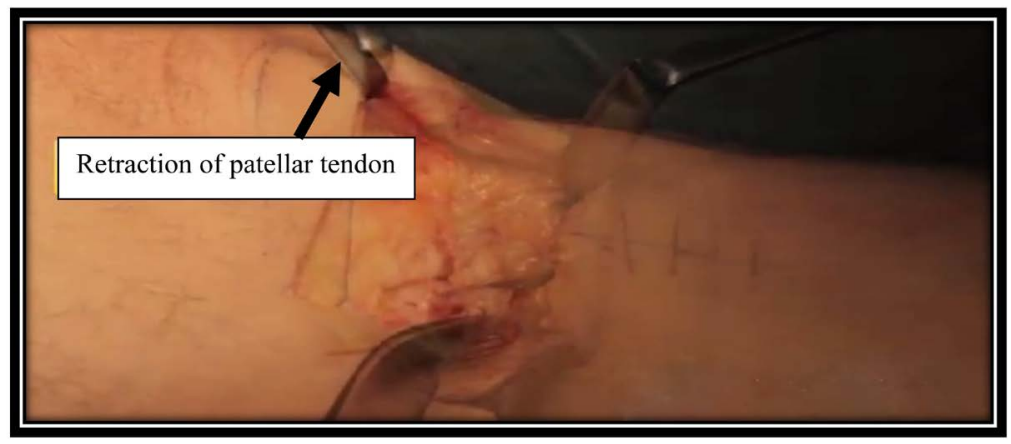

Figure 5. A retractor placed posteriorly to protect neurovascular bundle \& another one retracting patellar tendon.

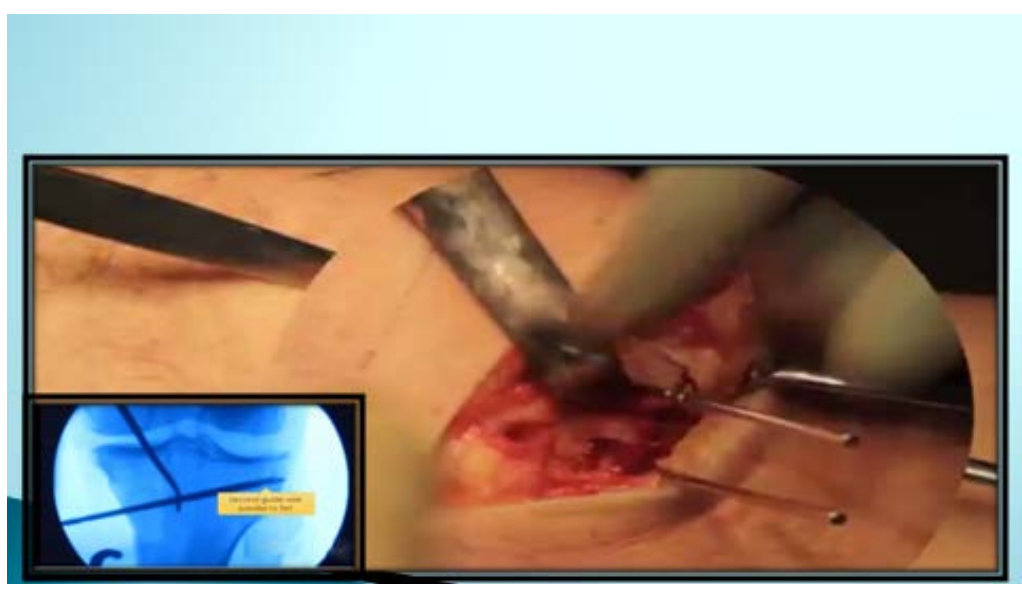

Figure 6. Under image control 2 parallel K-wires $(2 \mathrm{~mm})$ passed obliquely from medial border of tibia directed toward the tibio-fibuler joint.

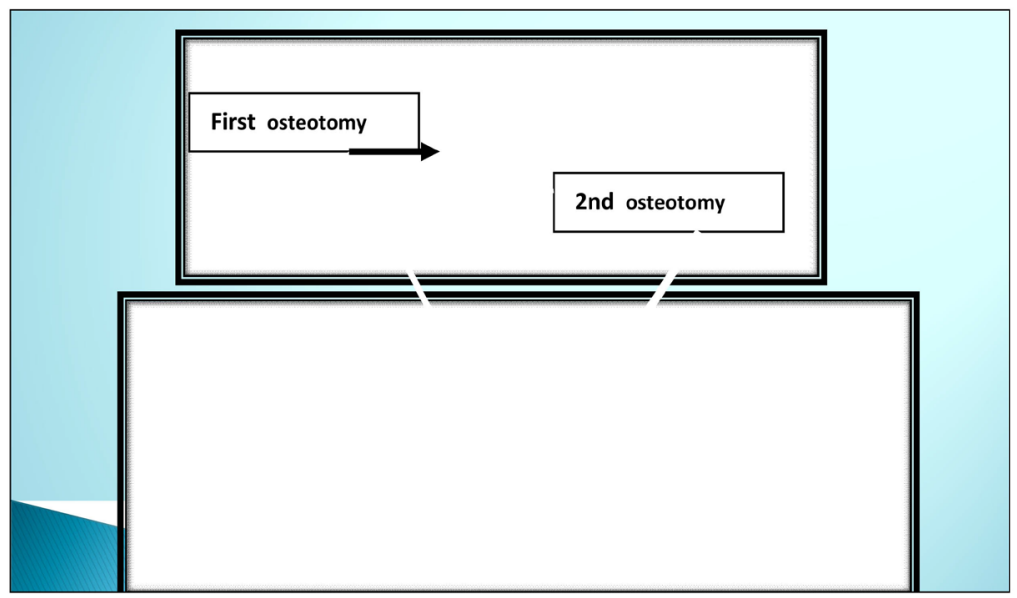

Figure 7. The first osteotomy cut run horizontally oblique targeting the tip of the fibular head, and the second osteotomy run oblique coronal at $130^{\circ}$ to the horizontal oblique osteotomy.

in the first distal hole below the osteotomy to increase stability by applying compression on the lateral hinge of the osteotomy. Unicortical screws were inserted in the remaining plate screw holes (Figure 9). 


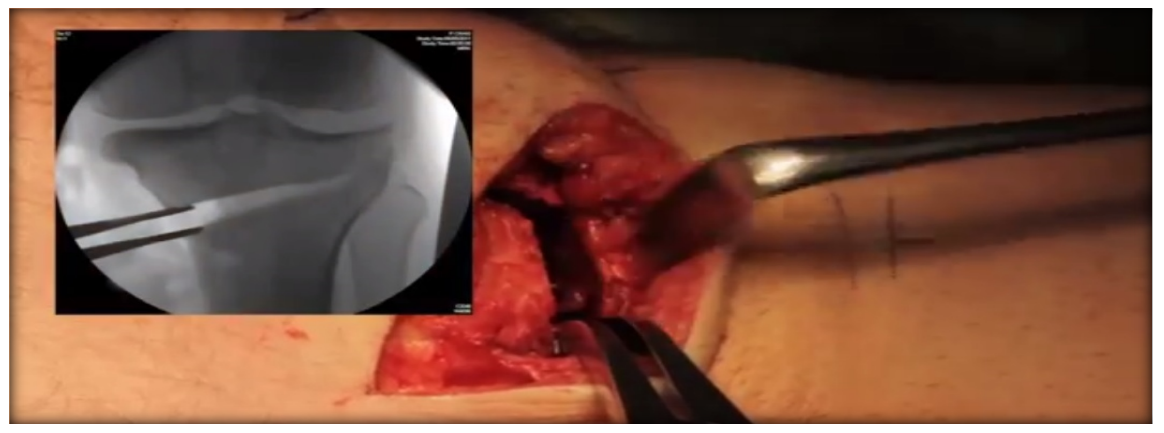

Figure 8. Laminar spreader inserted into osteotomy cut.

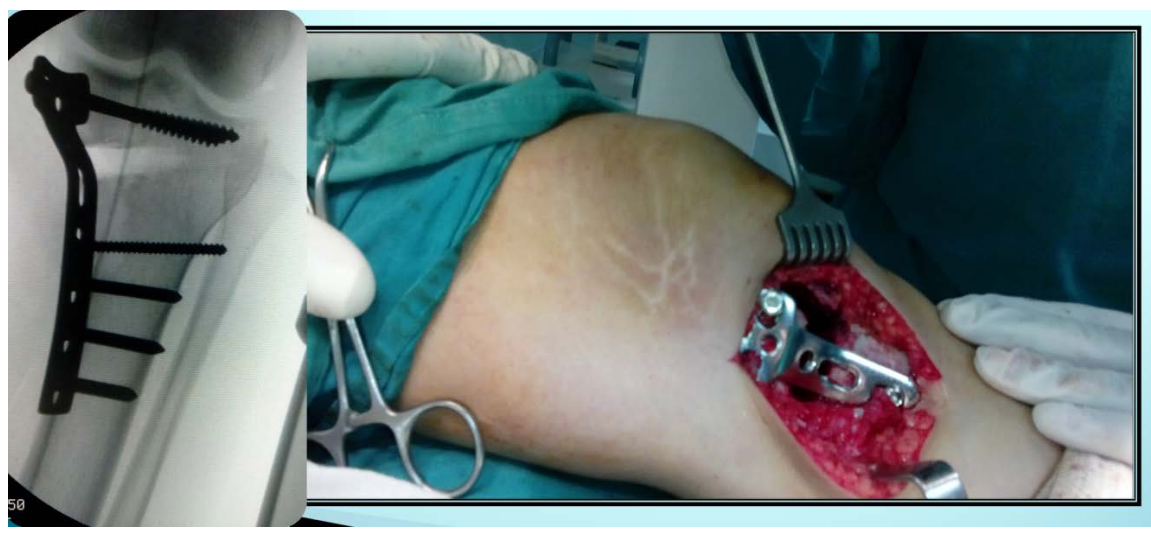

Figure 9. Low profile locking compression T-plate.

No drain applied and Closure of wound done then draping compression bandage applied over the whole leg.

\section{Postoperative care and follow-up:}

The post-operative protocol mainly depended on the rigidity of the fixation. The patients sent to physical therapy soon after surgery to try to decrease swelling and improve their overall muscle activation. This greatly helps with decreasing the need for narcotic pain medications. Non weight-bearing and active assisted exercises were allowed from second postoperative day in bed. Range of knee motion gradually increased till reach full range within 4 - 6 weeks. Partial weight-bearing allowed from 4 th week then full bearing without crutches when signs of bone union demonstrated both clinical (by absence of pain or local tenderness) and radiologically if bone healing of osteotomy demonstrated in two planes and no lysis zones or instability demonstrated.

\section{Results}

The patients were followed for average 28.5 months (18 - 35 months). No marked change in range of motion observed $\left(113^{\circ} \pm 3^{\circ}\right)$ preoperatively to $110^{\circ} \pm$ $6^{\circ}$ postoperatively) $\mathrm{p}>0.05$ however; Significant change of lysholm score was found $(45.5 \pm 21.7$ preoperatively, improved to $77.0 \pm 23.9$ out of 100 postoperatively) ( $\mathrm{p}<0.001)$. Knee society score recorded significant improvement following osteotomy as the average score improved from $51.19 \pm 11.82$ preoperative to 
$93.49 \pm 5.10$ postoperatively $(\mathrm{p}<0.001)$. The postoperative average corrected degree was $11.50 \pm 2.50^{\circ}\left(7^{\circ}-19^{\circ}\right)$ compared with the varus deformity before surgery. No cases with residual varus deformity $\left(\mathrm{mFTA}<0^{\circ}\right)$ after osteotomy and anatomical tibio-femoral angles from $0^{\circ}$ to $10^{\circ}$ valgus were achieved in all cases. Posterior tibial slope was maintained unchanged in 40 cases (83.3\%) while it increased in 8 patients $(16.7 \%)$ at the final follow-up. Preoperative posterior tibial slope was $8.04^{\circ} \pm 1.30^{\circ}$ and at last follow-up $9.15^{\circ} \pm 1.60^{\circ}$, respectively $(\mathrm{p}=$ 0.437 ) while the joint space distance increased from $4.05 \pm 1.30 \mathrm{~mm}$ preoperatively to $4.83 \pm 1.33 \mathrm{~mm}$ postoperatively $(\mathrm{p}<0.001)$. All patients had healing of the osteotomy with average time to complete bone union was $14.69 \pm 1.5$ weeks. There were no cases of delayed union or non-union. Four cases developed superficial wound infection which managed by daily dressing but no cases of deep infection developed. One case developed tendinitis of pes anserinus which responded well to antibiotic and nonsteroidal anti-inflammatory. None of the patients complained of pain that was directly related to prominence of the implant. All these minor complications completely resolved within 4 weeks from surgery. Hardware removal was performed in 25 patients after 2 years while 23 cases retained the implant. Changes of evaluation parameters are shown in Table 1.

Table 1. Evaluation of different variables.

\begin{tabular}{cccc}
\hline Variable & Preoperative & Postoperative & P value \\
\hline Range of motion & $113^{\circ} \pm 3^{\circ}$ & $110^{\circ} \pm 6^{\circ}$ & $\mathrm{P}>0.05$ \\
Varus deformity & $16.5^{\circ}\left(9^{\circ}-19^{\circ}\right)$ & $\begin{array}{c}\text { average corrected degree } \\
11.50^{\circ} \pm 2.50^{\circ}\left(7^{\circ}-19^{\circ}\right)\end{array}$ & \\
Posterior tibial slope & $8.04^{\circ} \pm 1.30^{\circ}$ & $9.15^{\circ} \pm 1.60^{\circ}$ & $\mathrm{p}=0.437$ \\
Joint space & $4.05 \pm 1.30 \mathrm{~mm}$ & $4.83 \pm 1.33 \mathrm{~mm}$ & $\mathrm{p}<0.001$ \\
Union time & & $14.69 \pm 1.5 \mathrm{weeks}$ & \\
Lysholm score & $45.5 \pm 21.7$ & $77.0 \pm 23.9$ & $\mathrm{p}<0.001$ \\
Knee society score & $51.19 \pm 11.82$ & $93.49 \pm 5.10$ & $\mathrm{p}<0.001$ \\
\hline
\end{tabular}

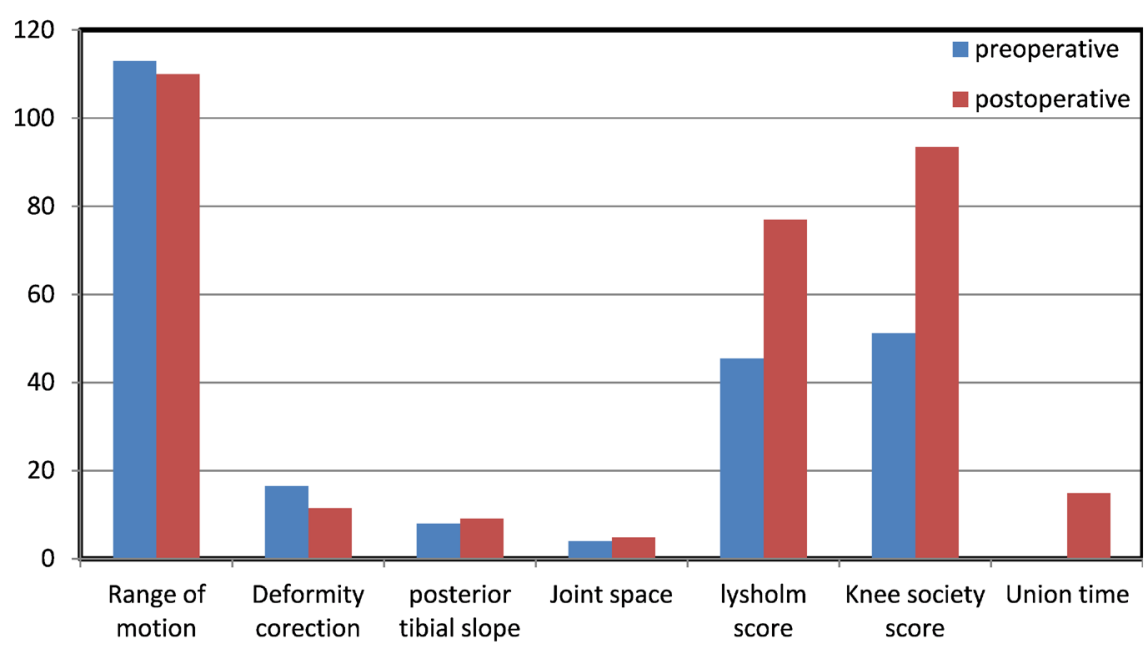




\section{Discussion}

Unicompartmental osteoarthritis with varus deformity constitutes a great challenge for orthopedic surgeons. The resultant limb malalignment allows more load to be distributed over the medial compartment. Many authors considered high tibial osteotomy as temporary surgery particularly in active patients who are not candidates for salvage arthroplasty [10]. Coventry (1965) [3] modified the procedures by making the osteotomy proximal to tibial tubercle so that the cancellous bone could heal rapidly, and early weight-bearing could be ensured. In recent years, a lot of articles concerned with open wedge high tibial osteotomy OWHTO have been published. However, optimization of surgical procedure is still a controversial issue. In a study involving 93 patients treated with medial OWHTO; the authors concluded that this technique was a suitable procedure for medial compartment gonarthrosis provided precise correction of malalignment achieved with the biologic healing of the osteotomy. None of the patients had symptoms of progression of the disease after 10 years [11]. The technique avoids several shortcomings associated with lateral closing wedge and dome osteotomies, including severence of tibiofibular joint, peroneal nerve injury, limb shortening, patella infera, and bone loss [12]. Nakamura et al., [13] compared 28 closing wedge osteotomies versus 40 dome osteotomies, with average varus alignment for each was $5.67^{\circ}$ and $1.45^{\circ}$, respectively recorded subjective satisfaction scores where $42.9 \%$ patients were unhappy with the closing wedge osteotomy compared with $57.5 \%$ with the dome osteotomy With the advent of biplanar technique in OWHTO Certain issues concerned including enhancement of osteotomy healing, sagittal inclination of tibial plateau, preservation of patellar height together with secondary alteration of the normal knee kinematics [2] [5] Particular concern in our present study directed toward the tibial plateau sagital inclination (posterior tibial slope PTS) which was maintained unchanged in 40 cases $(83.3 \%)$ while it increased in 8 cases $(16.7 \%)$ by less than $5^{\circ}$ and this was due to technical issue. To adequately maintain the posterior tibial slope, the opening ratio of the anterior to posterior gap should be 1 to 2 . Change of tibial slope would reduce translational forces and improve antero-posterior knee stability [14]. In a previous study by Hee-S. et al. [10], they concluded that PTSA changes of $<5^{\circ}$ were not clinically significant. Many biomechanical studies showed that increasing posterior slope aids in decreasing stress on the posterior cruciate ligament while decreasing the posterior slope decreases stress on the anterior cruciate ligament [15]. Also; patello-femoral arthritis is not a contraindication to HTO if modification of the technique done through coronal tibial correction combined with tibial tuberosity anteriorisation [16].

A prospective study on medial OWHTO by Schallberger et al. [17] of 54 patients with isolated medial compartment OA for a median of 16.5 years that were treated by either MOWHTO or lateral closing wedge osteotomy, and found $24 \%$ conversion to total knee arthroplasty. Moreover, there was no significant difference in score outcome and survival between open medial and closed lateral high 
tibia osteotomy.

Brouwer et al. [12] published results of a randomised study comparing the closing wedge technique to medial OWHTO fixed with the Puddu plate. Although, they did not see a loss of correction similar to our series, $60 \%$ of patients required the metalwork to be removed due to persistent discomfort and soft tissue irritation this is in contrast to our study in which none of the patients complained of pain related to prominence of the implant as we utilized low profile locked T-plate ( 21 cases) or low profile anatomical proximal tibial plate (27 cases). Also early weight bearing was gained in our study owing to use of rigid internal fixation and inherent stability of the osteotomy itself. Although Luites et al. [18] mentioned that there was no difference in weight bearing protocol between open and closed wedge HTO when fixed with locked compression plates. The locking plate used in our study provided an internal fixator with increased primary rotational stability in osteotomy site. No loss of correction, delayed union or non-union developed in our serial patients, and the patients were allowed to partially weight-bear early.

Different materials had been used to fill the osteotomy gap as autograft, allograft, cement spacers, and bone substitutes to enhance stability and promote bone healing. However, it has been reported that gaps less than $10 \mathrm{~mm}$ can be left unfilled for medial opening-wedge [19] Onodera et al. [20] studied on 38 patients undergoing MOWHTO using locking plate fixation and ceramic spacers. They found that post operative alignment and clinical outcome were comparable between hydroxyapatite (HAp) and betatricalcium phosphate (TCP), but TCP was significantly superior for oseoconductivity and bioabsorbability after 18 months. We did not use any material in our study to fill the osteotomy gap and in spite of that no cases developed delayed union or non-union.

In our prospective case series study final evaluation detected no marked change in range of motion. Also, significant improvement of the Knee Society score and lyshlom scoring scale detected. All cases had united with average time to complete bone union $14.69 \pm 1.5$ weeks. Staubli et al. [21] studied the bone healing using radiography after OWHTO without filling the osteotomy gap. They showed that healing starts from the lateral hinge and gradually progresses toward the medial aspect of tibia. Callus formation and ossification is visible three months after surgery. The new bone fills $75 \%$ of the gap 6 months after surgery. Almost $90 \%$ of the patients achieve full consolidation on radiography, CT scan, and MRI in one year.

Some advantages of Biplanar proximal tibial osteotomy technique used in our study are permission of correction of coronal and sagittal deformities with a single procedure, effectiveness biomechanically in both flexion and extension and better control of the correction. However; it is technically demanding procedure; need for at least 8-weeks period of none-weight bearing and risk of tibial fracture still warranted [22].

Some limitations of our study requiring consideration include small number of patients, short follow-up period which could not declare some shortcomings 
of long follow-up, lack of evaluation of tibial bone density with probability of osteoporosis that might affect functional outcomes and its affection on rigidity of implant fixation.

\section{Conclusion}

Biplanar opening high tibial osteotomy fixed with locking compression plate gives good functional results for symptomatic unicompartmental gonarthrosis with genu varum with relatively rapid bone healing and earlier rehabilitation. It allows correction of coronal and sagittal deformities with a single procedure and allows preservation of patellar height. Proper selection of patient is mandatory to get favorable results. Also this technique can be applied for medial compartment degeneration of the knee without deformity with good functional outcomes.

\section{Compliance with Ethical Standards}

- No benefits in any form have been received or will be received from a commercial party related directly or indirectly to the subject of this article.

- Ethical approval: This article does not contain any studies with animals.

- Informed consent was obtained from all individual participants included in the study according to the rules of the hospital research ethical committee.

- All procedures performed in our study were in accordance with the ethical standards of the institutional research committee and with the 1964 Helsinki declaration and its later amendments or comparable ethical standards.

\section{Disclosure}

Author contributions to the study and manuscript preparation include the following: Conception and design: Bahaa Korna; Acquisition of data: Mohamed Abdel-aal, Saied Kareem Analysis and interpretation of data: all authors; Drafting the article: all authors. Critically revising the article: all authors; Reviewed submitted version of manuscript: all authors.

\section{Conflicts of Interest}

The authors declare no conflicts of interest regarding the publication of this paper.

\section{References}

[1] Aglietti, P., Rinonapoli, E., Stringa, G. and Taviani, A. (1983) Tibial Osteotomy for the Varus Osteoarthritis. Clinical Orthopedic and Related Research, 176, 239-251.

[2] Langenbeck, B. (1854) Die subkutane osteotomie. Dtsch Klinik, 6, 327. (In German)

[3] Coventry, M.B. (1965) Osteotomy of the Upper Portion of the Tibia for Degenerative Arthritis of the Knee. The Journal of Bone and Joint Surgery. American, 47, 984-990. https://doi.org/10.2106/00004623-196547050-00008

[4] Fowler, P.J., Tan, J.L. and Brown, G.A. (2000) Medial Opening Wedge High Tibial Osteotomy: How I Do It? Operative Techniques in Sports Medicine, 8, 32-38. https://doi.org/10.1016/S1060-1872(00)80022-2 
[5] Kim, S.J., Mahajan, R.H., Park, K.Y., Kim, T.E. and Choi, W.J. (2007) Biplanar Medial Open-Wedge High Tibial Osteotomy for Medial Compartment Osteoarthritis of the Knee: A Novel Technique and Follow-Up. Operative Techniques in Orthopaedics, 17, 29-37. https://doi.org/10.1053/j.oto.2006.10.001

[6] Ahlbäck, S. (1968) Osteoarthrosis of the Knee. A Radiographic Investigation. Acta Radiologica: Diagnosis (Stockholm), Suppl 277, 7-72.

[7] Dugdale, T.W., Noyes, F.R. and Styer, D. (1992) Preoperative Planning for High Tibial Osteotomy: The Effect of Lateral Tibiofemoral Separation and Tibiofemoral Length. Clinical Orthopaedics and Related Research, 274, 248-264. https://doi.org/10.1097/00003086-199201000-00025

[8] Lysholm, J. and Gillquist, J. (1982) Evaluation of Knee Ligament Surgery Results with Special Emphasis on Use of a Scoring Scale. The American Journal of Sports Medicine, 10, 150-154. https://doi.org/10.1177/036354658201000306

[9] Fujisawa, Y., Masuhara, K. and Shiomi, S. (1979) The Effect of High Tibial Osteotomy on Osteoarthritis of the Knee. An Arthroscopic Study of 54 Knee Joints. Orthopedic Clinics of North America, 10, 585-608.

[10] Kyung, H.S., Lee, B.J., Kim, J.W. and Yoon, S.D. (2015) Biplanar Open Wedge High Tibial Osteotomy in the Medial Compartment Osteoarthritis of the Knee Joint: Comparison between the Aescula and TomoFix Plate. Clinics in Orthopedic Surgery, 7, 185-190.

[11] Herningou, P., Medevill, D., Debeyre, J. and Goutallier, D. (1987) Proximal Tibial Osteotomy with Varus Deformity: A Ten to Thirteen Year Follow-Up Study. The Journal of Bone and Joint Surgery. American, 69, 332-354. https://doi.org/10.2106/00004623-198769030-00005

[12] Brouwer, R.W., Bierma-Zeinstra, S.M., van Raaij, T.M. and Verhaar, J.A. (2006) Osteotomy for Medial Compartment Arthritis of the Knee Using a Closing Wedge or an Opening Wedge Controlled by a Puddu Plate. A 1-Year Randomised, Controlled Study. The Journal of Bone and Joint Surgery. British, 88, 1454-1459. https://doi.org/10.1302/0301-620X.88B11.17743

[13] Nakamura, E., Mizuta, H., Kudo, S., Takagi, K. and Sakamoto, K. (2001) Open-Wedge Osteotomy of the Proximal Tibial Hemicallotasis. The Journal of Bone and Joint Surgery. British, 83, 1111-1115. https://doi.org/10.1302/0301-620X.83B8.11993

[14] Marti, C.B., Gautier, E., Wachtl, S.W. and Jakob, R.P. (2004) Accuracy of Frontal and Sagittal Plane Correction in Open-Wedge High Tibial Osteotomy. Arthroscopy, 20, 366-372. https://doi.org/10.1016/j.arthro.2004.01.024

[15] El-Azab, H., Halawa, A., Anetzberger, H. and Hinterwimmer, S. (2008) The Effect of Closed- and Open-Wedge High Tibial Osteotomy on Tibial Slope: A Retrospective radiological Review of 120 Cased. The Journal of Bone and Joint Surgery. British, 90, 1193-1197. https://doi.org/10.1302/0301-620X.90B9.20688

[16] Abdel Megied, W.S., Mahran, M.A., Thakeb, M.F., Amr, A.K. and Elbatrawy, Y. (2010) The New "Dual Osteotomy": Combined Open Wedge and Tibial Tuberosity Anteriorisation Osteotomies. International Orthopaedics, 2, 231-237.

[17] Schallberger, A., Jacobi, M., Wahl, P., Maestretti, G. and Jakob, R.P. (2011) High Tibial Valgus Osteotomy in Unicompartmental Medial Osteoarthritis of the Knee: A Retrospective Follow-Up Study over 13-21 Years. Knee Surgery, Sports Traumatology, Arthroscopy, 19, 122-127. https://doi.org/10.1007/s00167-010-1256-4

[18] Luites, J.W.H., van Heerwaarden, R., Valstar, E.R. and Wymenga, A.B. (2006) Stability of Open vs. Closed Wedge High Tibial Osteotomy Measured with Roentegen Stereophotogrammetric Analysis in a Randomised Clinical Trial. ESSKA Congress. 
[19] Pape, D., Lorbach, O., Schmitz, C., et al. (2010) Effect of a Biplanar Osteotomy on Primary Stability Following High Tibial Osteotomy: A Biomechanical Cadaver Study. Knee Surgery, Sports Traumatology, Arthroscopy, 18, 204-211. https://doi.org/10.1007/s00167-009-0929-3

[20] Onodera, J., Kondo, E., Omizu, N., et al. (2014) Beta-Tricalcium Phosphate Shows Superior Absorption Rate and Osteoconductivity Compared to Hydroxyapatite in Open-Wedge High Tibial Osteotomy. Knee Surgery, Sports Traumatology, Arthroscopy, 22, 2763-2770. https://doi.org/10.1007/s00167-013-2681-y

[21] Staubli, A.E. and Jacob, H.A. (2010) Evolution of Open-Wedge High-Tibial Osteotomy: Experience with a Special Angular Stable Device for Internal Fixation without Interposition Material. International Orthopaedics, 34, 167-172.

https://doi.org/10.1007/s00264-009-0902-2

[22] Hopwood, S., Khan, W. and Agarwal, S. (2016) The Biplanar Open Wedge High Tibial Osteotomy Preserving the Tibial Tubercle. Journal of Orthopaedic Science, 21, 786-790. 